\title{
RAPID IDENTIFICATION OF ABIOTIC STRESS (FROST) IN IN-FILED MAIZE CROP USING UAV REMOTE SENSING
}

\author{
Jonali Goswami ${ }^{1, *}$, Vikas Sharma ${ }^{1}$, Burhan U Chaudhury ${ }^{2}$, PLN Raju ${ }^{1}$ \\ ${ }^{1}$ North Eastern Space Applications Centre, Department of Space, Government of India, Meghalaya, India - (jonali.goswami, \\ vikashsharmamy, plnraju)@gmail.com \\ ${ }^{2}$ ICAR Research Complex for NEH Region, Umiam, Ri-Bhoi district, Meghalaya - 79103, India. - (burhan3i@yahoo.com)
}

Commission III, WG III/10

KEY WORDS: Abiotic stress, Maize crop, Remote Sensing, UAV, Machine Learning, Classification.

\begin{abstract}
:
Stress in the crop not only decreases the production but can also have devastating consequences for farmers whose life depends upon the healthy crops. In recent time (January 2018) a such abiotic stress event (hoar frost) was experienced at ICAR research complex experimental filed, Ri-Bhoi district of Meghalaya on standing Maize crop. Therefore, remote sensing (Multispectral UAVUnmanned Aerial Vehicle) technology were used to detect the effect of frost on in-filed Maize crop. Two set of multispectral data (before frost and after frost) with four advanced machine learning techniques viz. Random Forest (RF), Random Committee (RC), Support Vector Machine (SVM) and Artificial Neural Network were employed for detection of stress free crop and stressed crop due to frost. Results revealed that all the four methods of classification could able to identify / detect stress-free vs. stressed crops at satisfactory level. However, among the classifiers RF achieved relatively higher overall accuracy $(\mathrm{OA}=86.47 \%)$ with Kappa Indexanalysis $(\mathrm{KIA}=0.80)$ and found very cost effective in context of computational cost (time complexity $=0.08$ Seconds) to train the model. In addition, we have also recorded the area of each classes and found that after frost stress-free area (36.01\% of all over filed) is decreased by $11 \%$ in comparison of before frost $(25.036 \%$ of all over filed). Based on the results we can suggest that the RF ensemble classification method can be used for further other crop classification in order to estimate the yield, detect the condition, monitoring the health etc.
\end{abstract}

\section{INTRODUCTION}

Crop growth sensitivity to temperature, global increase and higher extremes of temperature represent a threat to crops and may cause yield changes and production losses (Ruiz-Ramos et al., 2011). Quantification of agriculture risks is very important in understanding the extent of risk and planning for its effective mitigation. Therefore, weather related risk in agriculture has get great attention of researchers and practitioners in last two decades as the occurrence of uncertain extreme climatic events (drought, flood, hailstorm, and frost etc.) increasing rapidly (Berthet et al., 2011; Changnon et al., 2009; Gobin et al., 2012 ;SaaRequejo et al., 2011). Frost is such unpredictable event that can occur at any point of time and causes partial and full damage to the crop (GOBIN et al., 2013). Frost related crop damage is relatively rare, particularly in India. However, with the changing climate, the last 20 years have seen a significant increase in the occurrences of frost. (Grewal and Singh, 1980). Frosts can be extremely devastating as they are sudden impact. One frost event can ruin entire crops and yields by causing plant floret sterility, affecting the stem development and causing tiller death (K. Brun-Laguna et al, 2016). Most frost events occur during clear and calm nights. Rapid estimation of frost damage to crops on a spatial basis would allow for timely management decisions to reduce the economic impact of frost events (Perry at al., 2017).

Maize (Zea mays L.) is the third major cereals in world after rice and wheat. In 2015-16 India has sown maize crop in the 8.69 million hectare and crop yield 21.81 million tons. Maize being a thermophilic species sensitive to chilling temperature (below $10^{\circ} \mathrm{C}$ ) resulting in yield loss or crop failure.Maize is sensitive to frost in all phases of its growth cycle except as dry seed (Miedema, P., 1982). In January 2002-03 due to cold wave early sown maize crop was adversely affected in different region of India and loss was up to $70-80 \%$ in some. According to report of ICAR 2002-03 in Shillong valley (Study area) cold wave severity destroyed the crop for an extent of $16 \%$ to $100 \%$.

Remote Sensing (RS) is a very efficient technology in terms of cost benefit as it allows large areas of homogeneous data and performs multi-temporal analysis. RS data can be gathered through space borne satellites, cameras flown on light aircraft or unmanned aerial vehicles (UAV), and handheld (proximal) sensors. The spectral information captured in the thermal infrared is possible to estimate the surface temperature, which allows for example to study the occurrence of frost (Perry et al., 2017).

Image classification is a popular and effective tool that can be used to identify and study most relevant information in RS data. In any RS research, the decision-making way mainly rely on the efficiency of the classification framework (Radhika and Varadarajan, 2016). In classification of high resolution RS data machine learning based classification algorithms have shown excellent performance on many benchmark datasets. Various machine learning algorithms like Support Vector Machine (SVM), Random Forest (RF), Random Committee (RC), and Artificial Neural network (ANN) has been successfully solved various problems through classification.

Macedo-Cruz et al. 2011 classified digital camera images (red, green, blue) taken 15 days after the last frost on an oat crop into classes of healthy, frost damaged (yellow leaves), intermediate damage, and shade.

*Corresponding author 
The optimal spectral regions for detecting frost damage still need to be defined as little is known about the structural or biochemical changes induced by frost that may be detected as changes in reflectance signatures. Flower et al. 2014 found that when compared with the leaves of non-frosted wheat plants, leaves of those that had been subjected to a frost treatment exhibited changes in reflectance between 500-680 nm and 730$740 \mathrm{~nm}$. Wu et al. 2012 acquired spectral imagery (400-1000 $\mathrm{nm}$ ) for potted wheat seedlings on 1-2 week intervals from seedling emergence to the occurrence of freezing injury. In 1997 C. JURGENS proposed modified normalized difference vegetation index (mNDVI) index to determine frost damages in wheat based on Landsat TM data (Brun-Laguna et al., 2016). The proposed system consists of an IoT-based network architecture in charge of the acquisition of weather attributes directly taken from the crop field. Perry et al. 2017 used hyperspectral reflectance and active light fluorescence for detecting frost damage in wheat during its reproductive phase and found that the hyperspectral reflectance data appeared variable in response. Major causes for declining crop productivity in agriculture when it damages the crop at peak season. Extreme weather events like frost, already a significant challenge for grain producers, are predicted to increase under future climate scenarios. A hoar frost has been experienced at Shillong valley, north eastern part of India on $10^{\text {th }}$ of January 2018. Therefore, quick damage assessment has been carried out on standing winter maize crop using UAV based remote sensing and machine learning techniques.

\section{METHODOLOGY}

Four channels multispectral UAV data were acquired from the experimental field on 80-meter height. After the initial preprocessing (i.e. described in next section) of data a combination of all four channels images (stacked images) were used for classification process.

After classification, accuracy assessment was also done using the well versed accuracy parameters i.e. overall accuracy, kappa index analysis and computational cost.

As described in earlier sections four advanced different machine learning techniques were employed to found the effect of frost on Maize crop using classification. The main objective of using various variant of classifiers to see their performance and significance to detecting the changes in crop health before and after frost. The detailed information about the all four machine learning techniques are given in next sub sections.

\subsection{Random Forest}

$\mathrm{RF}$ is a very popular and effective ensemble method that follows the principle of bagging. It uses the decision trees as a base classifier and create the $n$ number of different bags for training the base classifiers. In RF randomization in the training data is not only achieved by the creating diversity in training sample but it also creates the randomization in feature space using information gain, gini index, and gain ration. Gini index is responsible for best feature selection to grow a particular tree. It calculates the error based on the misclassified pixel and try to find the best feature with minimal error. Based on the best feature the corresponding tree has been grown. The final prediction of RF is based on the majority of votes (Breiman, 2001).

Furthermore, attribute bagging was used to reduce the misclassification rate and to increase performance of classifier. The best set of features and $\mathrm{k}$ decision trees are opted after repeating this process for $\mathrm{k}$ times. Now RF with $\mathrm{k}$ - random DTs is observed. Here, 100 number of $\mathrm{C} 4.5$ decision tree was used as a base classifier. The main concern to using the RF is to define the optimal number of decision trees. Therefore, here hundred number of random trees were used for classification purpose.

\subsection{Random Committee}

Random committee is a meta ensemble method for combining the weak learners in order to increasing the productivity of the classification model. It constructs an ensemble of base classifiers and produce their results as an averages of all. The all weak learners are train with the same training data but different random number seed is used to create the diversity in training data (Witten and Frank, 2005).

In this classification process 100 number of $\mathrm{C} 4.5$ decision tree was initialised as a weak learner

\subsection{Support Vector Machine (SVM)}

SVM being non parametric classifier uses the hyperplane to classify the instances. Based on the support vectors, hyperplane boundary between the different classes is realized in the SVM classifier. The ultimate goal of SVM is to maximize the boundary between two classes in order to select the optimal hyperplane boundary. Various kernels functions (Like Linear, polynomial, Radial Basis Function, Sigmoid) are being used to decrease the computational cost of SVM by using the inner product of 2 transformed data vectors in the feature space (Cortes and Vapnik, 1996).

For an instance, $D$ is the set of all input data, and $\mathrm{X}_{\mathrm{n}}$ is the input space with target class $\mathrm{Y}_{\mathrm{I}}$ then-

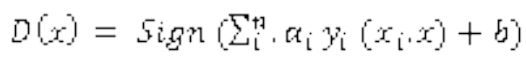

The main advantage of SVM is it can be trained with minimal number of training samples. On the other hand, the biggest limitation of SVM is to selecting the optimal kernel and its parameters. However, in remote sensing data classificaiotn a number of studies suggested that the polynomial kernel can perform better in comparison of all other above mentioned kernels. Therefore, in this experiment SVM with polynomial kernel was realised to classify the multiclass data (Rawat et al, 2017; Sharma et al., 2016; Sharma et al., 2018).

\subsection{Artificial Neural Network (ANNs)}

ANN are noteworthy for being adaptive as they can modify themselves based on the initial training and subsequent iterations. An ANN model usually involves 3 layers in their architecture i.e. I.) input layer II) hidden layer and III) Output Layer. The all layers of an ANN are highly interconnected with each other. Input layer which receives the input from training data applied the weights on it and forward it to the computation layer (hidden layer). In hidden layer neurons are available to process the input with the help of non linearactivation function. A bias can play the critical role in order to minimizing the cost function of an ANN model. Multiple hidden layers can be used to train the ANN but it requires more computational power and high end computation facility. In contrast of it, large number of hidden layers can be helpful to increase the classification accuracy. On the other hand, ANN generally suffers from overfitting when training goes too long and they also require highly expert knowledge in the training phase (Rollet, R., 1998, Huang, 2006). 
In this research work three number of hidden layers were realized with backpropagation technique to train the ANN model.

\section{STUDY AREA AND DATA}

The experimental research field of ICAR-NEH region is situated at Umiam,Ri-Bhoi district atIndian states of Meghalaya is considered as the study area for this experiment.

For DST funded ongoing project the Maize was growing in the experimental field. after frost the UAV data with ground truth has been collected from the same field in the observation of experts. A total number of 964 and 1017 samples were identified for the training and testing of the classifiers respectively.

\subsection{Unmanned Aerial Vehicles (UAV's)}

UAVs have recently gain momentum in agricultural data collection methods as it provides very high resolution images with many new and unique possibilities, as well as new and unique challenges [24]. In this experiment, a light weight hexacopter DJI Matrix 600 was employed for the periodic field survey. This model of UAV can fly 45-50 minutes continuously within a radius of $5 \mathrm{~km}$ range. A parrot Sequoia multispectral camera mounted on UAV has been used to collect the data. Green (550nm), Red (690nm), Red Edge (735nm) and NIR $(790 \mathrm{~nm})$ are the four channels in which the multispectral camera records the reflected energy in central wavelength.

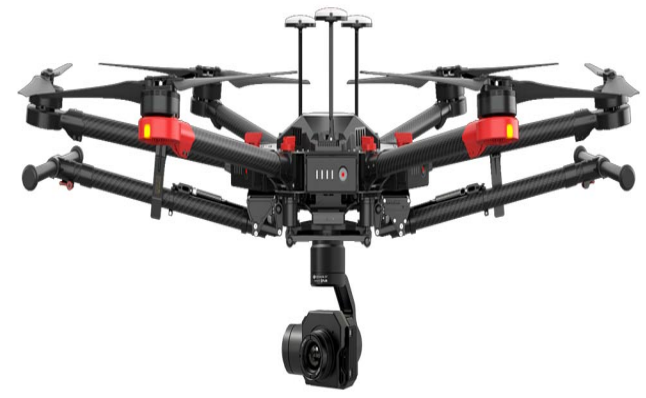

Figure 1. UAV model of DJI Thermal Matrice 600.

(Source: https://www.dji.com/matrice600)

In UAV the DJI flight planner app was used for automatic flight planning. The data collected through multispectral camera has been processed using Pix4D software. Around 700-900 images per field visit were taken. The Geo-location was taken for the accuracy of on-board GPS. After initial processing the generation of 3D point cloud and mesh was done. This 3D point cloud was used to make the digital terrain model, digital surface model and orthomosaic. Then different separate bands wereadded to create a single multispectral image. This multispectral image then further used for classification purpose.

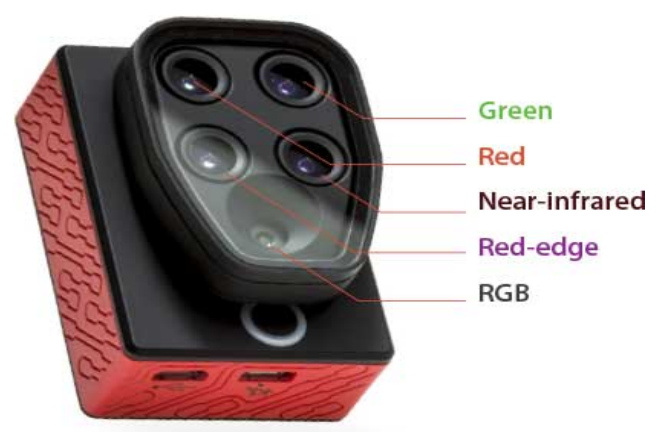

Figure 2. Parrot Sequoia Multispectral sensor

(Source: https://www.parrot.com/business-solutions-us/parrotprofessional/parrot-sequoia)

\subsection{Information about the train and test dataset}

Based on the field architecture and expert views a total four number of classes were identified to detect the health of crop. Train and test data for each class has been recorded based on the ground truth. The detailed information about the classes with train and test data can be found in below given table 1. The same train and test data was used to train the all classifiers.

\begin{tabular}{|l|l|l|l|}
\hline S. no. & Classes Name & $\begin{array}{l}\text { Number of } \\
\text { Training } \\
\text { Sample }\end{array}$ & $\begin{array}{l}\text { Number of } \\
\text { Test Sample }\end{array}$ \\
\hline 1 & Healthy & 278 & 251 \\
\hline 2 & Stressed & 255 & 244 \\
\hline 3 & Weeds & 193 & 366 \\
\hline 4 & Non-cropped/border & 238 & 156 \\
\hline & Total & 964 & 1017 \\
\hline
\end{tabular}

Table 1. Number of training and tested samples collected from maize fields for UAV image classification

\section{RESULTS AND DISCUSSION}

Total 2 set of 4 bands multispectral images were used in this experiment i.e. before frost and after frost. After initial preprocessing (illustrated in earlier section) training data was used for training the model. The same training data were used to classify all 2 sets of images in order to detect the health of crop in both cases (before and after frost). The open source high level python programming language has been used to perform the classification task.

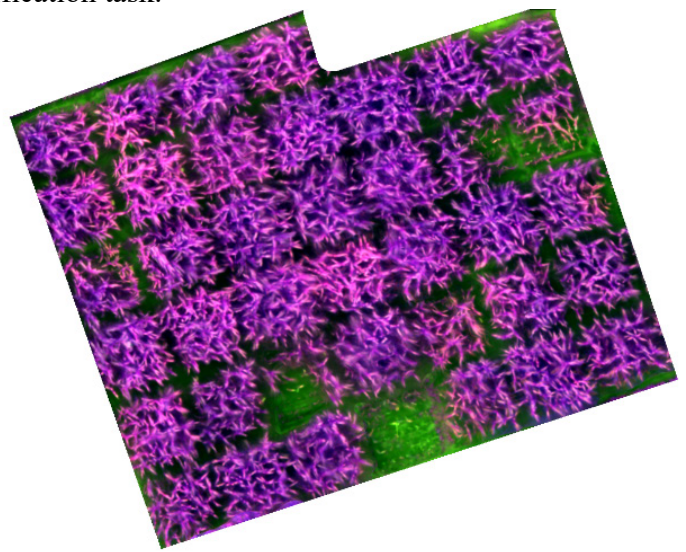

Figure 3. Multispectral image of experimental field. 
Results revealed that all the four methods of classification could able to identify / detect stress-free vs. stressed crops at satisfactory level. Among the classifiers, RF registered relatively higher overall accuracy (Accuracy $=86.47 \%$, Kappa Index analysis $=0.80$ ) with shorter training time (0.08 Seconds) than other three classifiers. Artificial neural network took longest training time (02 minutes) with low overall accuracy $(80 \%)$ and kappa index analysis (0.74) than RC (overall accuracy $=85.39$, kappa index analysis $=0.77$ and time complexity $=0.15$ minutes) and SVM (overall accuracy $=82.33$, kappa index analysis $=0.76$, and time complexity $=01: 43$ minutes) [Table 2].In classified image it is clearly observed that frost make an impact on crop health. The yellow area (stressed) in the classified image is increased drastically after the frost. The classified images before and after frost can be found in Figure 4.

\begin{tabular}{|c|c|c|c|c|}
\hline S. No. & Classifiers & $\begin{array}{c}\text { Kappa } \\
\text { Index } \\
\text { Analysis }\end{array}$ & $\begin{array}{c}\text { Overall } \\
\text { Accuracy }\end{array}$ & $\begin{array}{c}\text { Training } \\
\text { time (in } \\
\text { minutes) }\end{array}$ \\
\hline 01 & SVMs & 0.76 & 82.83 & $01: 43$ \\
\hline 02 & RF's $^{\prime}$ & 0.80 & 86.47 & $0: 08$ \\
\hline 03 & RC's $^{\prime}$ & 0.77 & 85.39 & $0: 15$ \\
\hline 04 & ANN & 0.74 & 80.0 & $02: 00$ \\
\hline
\end{tabular}

Table 2: Accuracy of the advanced machine learning classifiers

In this study we have also recorded the area covered by different above mentioned classes in order to see the area is covered by a particular class is decreased or increased. In the initial stages of crop growth (30 days old), nearly $29 \%$ area had healthy stress free crops which with the advancement of growth stages (on 60 days old), reached to $36 \%$ of the total area (600 sq. m). Generally, maize reaches its peak vegetative stages at 90 days (falling in January end) and that time, canopy ground coverage also increases significantly. However, due to severe frost occurrence at peak vegetative stages of growth (10th January, 2018), frost induced injury resulted in partial failure/ death of crops in many plots. As a result, during March-2018 (120 days old), health cropped area decreased by $11 \%$ over December-2017 (before frost occurrence). Similarly, area under stressed crops increased from $7.9 \%$ in November 2017 to over $17 \%$ in December and after frost injury, many plots experienced complete crop failure. As a result, post-frost stressed crop area increased further $(>17 \%$ with partial stress while $>39 \%$ area with complete crop failures). This was evident from the significant increase in non-cropped area from $12.77 \%$ in December- 2017 to $51.74 \%$ in march-2018 (Table 3).

\begin{tabular}{|l|l|l|l|}
\hline \multirow{2}{*}{ Class Name } & \multicolumn{3}{|c|}{ Area in percentage } \\
\cline { 2 - 4 } & $\begin{array}{l}\text { November } \\
2017\end{array}$ & $\begin{array}{l}\text { December } \\
2017\end{array}$ & $\begin{array}{l}\text { March } \\
2018\end{array}$ \\
\hline Healthy & 28.838 & 36.01 & 25.036 \\
\hline Stressed & 7.944 & 17.55 & 17.497 \\
\hline Weed & 28.531 & 33.67 & 5.728 \\
\hline $\begin{array}{l}\text { Non- } \\
\text { cropped/border }\end{array}$ & 34.687 & 12.77 & 51.739 \\
\hline
\end{tabular}

Table 3. The percentage wise calculation of area for under different classes.

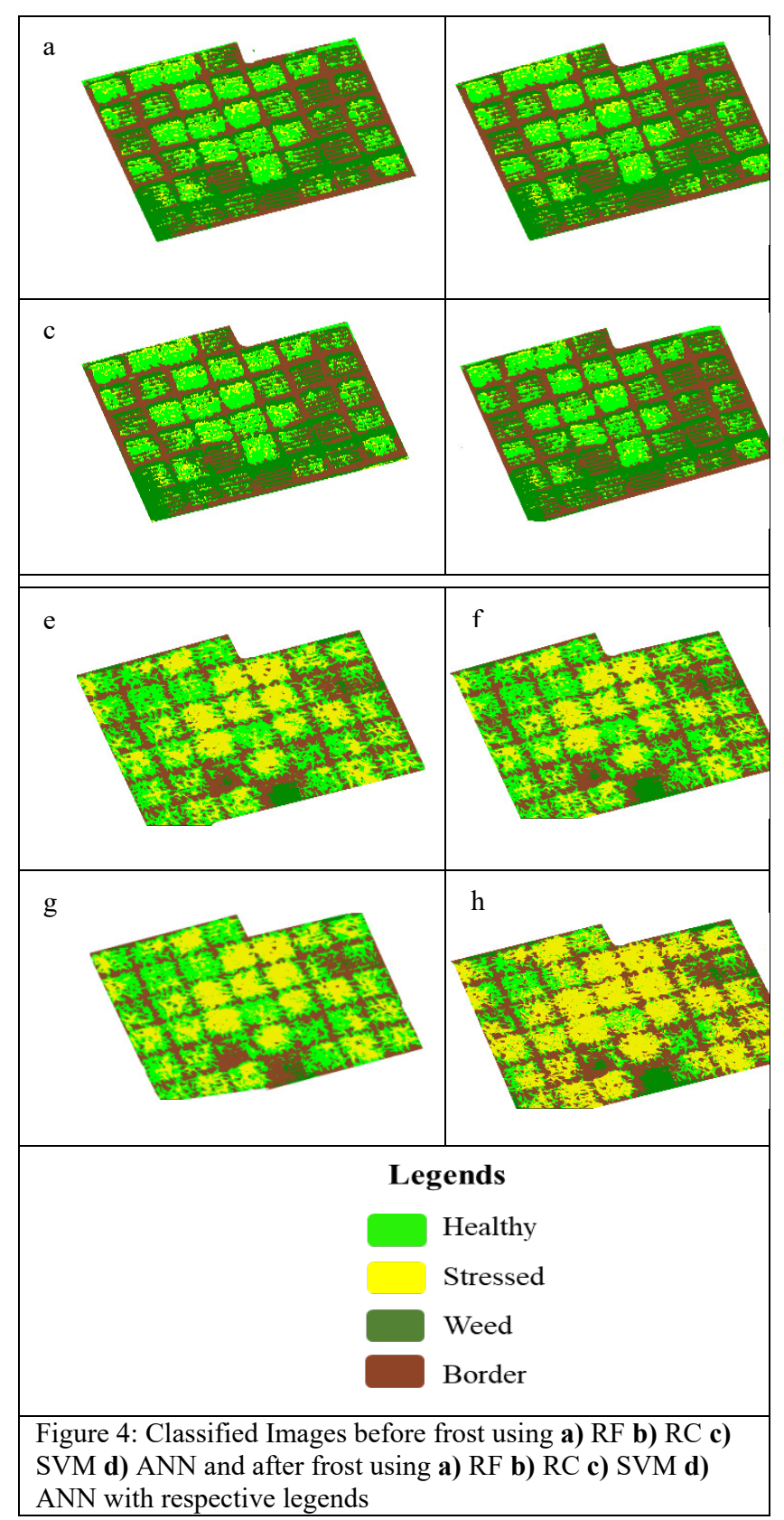

\section{CONCLUSION AND FUTURE SCOPE}

All four machine learning techniques has been performed quite better and able to detect the impact of frost on crop as illustrated in results and discussion section. Based on our results we can suggest that RF can be a better option sfor detecting the health of crop or to detect the impact of frost on crop. However, the abiotic stress due to nutrient deficiency can be assessed in coming time using these classifiers with the hyperspectral data. In technological enhancement deep learning also can be explored with a huge number of training data.

\section{ACKNOWLEDGEMENTS}

Authors would like to acknowledge the support of Department of Science and Technology (DST), Govt. of India to funding this project to Indian Council of Agricultural Research for NEH 
region (ICAR-NEH). Authors also acknowledge the supports of North Eastern Space Applications Centre (NESAC), dept. of Space, Govt. of India, for providing essential scientific equipment's and environment for smooth execution of this research work.

\section{REFERENCES}

Berthet, C., Dessens, J., and Sanchez, J. L.: Regional and yearly variations of hail frequency and intensity in France, Atmos. Res., 100, 391-400, doi:10.1016/j.atmosres.2010.10.008, 2011.

Breiman, L. (2001). Machine Learning, 45(1), pp. 5-32. https://doi.org/10.1023/A:1010933404324.

C. JUÈ RGENS " The modified normalized divergence vegetation index (mNDVI) a new index to determine frost damages in agriculture based on Landsat TM data ", international journal of remote sensing, 1997, vol. 18, no. 17, pp.3583-3594.

Changnon, S.: Increasing major hail losses in the US, Climatic Change, 96, 161-166, 2009.

Cortes, C. and V. Vapnik. (1996). Support-Vector Networks, Machine Learning, 20(3),273-297. doi:10.1007.BF00994018.

Eileen M. Perry A,C, James G. Nuttall B, Ashley J. WallaceB, and Glenn J. Fitzgerald B," In-field methods for rapid detection of frost damage in Australian dryland wheat during the reproductiveand grain-filling phase", Crop \& Pasture Science, 2017, 68, 516-526, http://dx.doi.org/10.1071/CP17135.

Flower K, Boruss B, Nansen C, Jones H, Thompson S, Lacoste C, Murphy M (2014) Proof of concept: remote sensing frostedinduced stress in wheat paddocks. Grains Research and Development Corporation, Canberra.

Gobin, A.: Impact of heat and drought stress on arable crop production in Belgium, Nat. Hazards Earth Syst. Sci., 12, 19111922, doi:10.5194/nhess-12-1911-2012, 2012.

Gobin, A., Tarquis, A. M., and Dalezios, N. R.: Preface "Weather related hazards and risks in agriculture", Nat. Hazards Earth Syst. Sci., 13, 2599-2603, doi:10.5194/nhess-13-25992013, 2013

Grewal, J.S. \& Singh, S.N. Plant Soil (1980) 57: 105. https://doi.org/10.1007/BF02139646

Huang, Guang-Bin; Zhu, Qin-Yu; Siew, Chee-Kheong (2006). "Extreme learning machine: theory and applications". Neurocomputing. 70 (1): 489 501. 10.1.1.217.3692. doi:10.1016/j.neucom.2005.12.126.

Macedo-Cruz A, Pajares G, Santos M, Villegas-Romero, "Digital image sensor-based assessment of the status of oat (Avenasativa L.) crops after frost damage", Sensors (Basel). 2011;11(6):6015-36. doi: 10.3390/s110606015.

K. Brun-Laguna et al., "A Demo of the PEACH IoT-Based Frost Event Prediction System for Precision Agriculture," 2016, 13th Annual IEEE International Conference on Sensing,
Communication, and Networking (SECON), London, 2016, pp. 1-3. doi: 10.1109/SAHCN.2016.7732963.

K. Radhika and. S. Varadarajan, "A TUTORIAL ON CLASSIFICATION OF REMOTE SENSING DATA", International Research Journal of Engineering and Technology (IRJET), vol 03 issue : 08 Aug 2016, pp 881-885.

Miedema, P. (1982). The Effects of Low Temperature on Zea mays. Adv. Agron.. 35. 93-128. 10.1016/S00652113(08)60322-3

Rawat, U., V. Sharma, D. Chutia, N. Nishant, U. Baruah, U. and P.L.N. Raju. (2018). Random forest versus rotation forest - A comparative assessment towards classification of satellite data, 5th IEEE International Conference on Computing for Sustainable Global Development, 14th - 16th March, Proceedings of the 12th INDIACom; pp. 1732-1736. INDIACom-2018; IEEE Conference ID: 42835

Ruiz-Ramos, M., Sánchez, E., Gallardo, C. and Mínguez, M. I.: Impacts of projected maximum temperature extremes for C21 by an ensemble of regional climate models on cereal cropping systems in the Iberian Peninsula, Nat. Hazards Earth Syst. Sci., 11, 3275-3291, doi:10.5194/nhess-11-3275-2011, 2011.

Rollet, R., Benie, G.B., Li, W., Wang, S. \&amp; Boucher, J.M. Image Classification Algorithm based on the RBF Neural Network and K-means, International Journal of Remote Sensing, 19, 3003--3009, 1998.

SaaRequejo, A., García Moreno, R., Díaz Alvarez, M. C., Burgaz, F., and Tarquis, M.: Analysis of hail damages and temperature series for peninsular Spain, Nat. Hazards Earth Syst. Sci.,11, 3415- 3422, doi:10.5194/nhess-11-3415-2011, 2011.

Sharma, V., D. Baruah, D. Chutia, P.L.N. Raju. andD.K. Bhattacharya. (2016). An assessment of support vector machine kernel parameters using remotely sensed satellite data, IEEE International Conference on Recent Trends in Electronics, Information \& Communication Technology (RTEICT), 15671570. doi:10.1109/RTEICT.2016.7808096.

Sharma, V., N. Choudhury, R. Mandal, D. Chutia, U. Baruah. R. Kumar. (2018).Anempirical study on traditional classifiers for remotely sensed satellite data classification,5th IEEE International Conference on Computing for Sustainable Global Development, 14th - 16th March, Proceedings of the 12th INDIACom; pp. 2032-2036. INDIACom-2018; IEEE Conference ID: 42835.

Witten, I.H., and E. Frank. (2005). Data Mining: Practical machine learning tools and techniques, San Francisco: CA: ELSEVIER, 2nd ed., chapter.7 and 10.

Wu Q, Zhu D, Wang C, Ma Z, Wang J (2012) Diagnosis of freezing stress in wheat seedlings using hyperspectral imaging. Biosystems Engineering 112, 253-260. 